\title{
Evaluation Model for Protocol Conformance of BDS D1 Navigation Messages
}

\author{
Menglan Wang ${ }^{1}$, Zhicheng $\mathrm{Lv}^{1,}{ }^{*}$, Jing Peng ${ }^{1}$, Weihua $\mathrm{Mou}^{1}$, and Gang $\mathrm{Ou}^{1}$ \\ ${ }^{1}$ Engineering Research Center for Position, Navigation and Time, College of Electronic Science, National University of Defense \\ Technology, Deya Road 109, Changsha, China
}

\begin{abstract}
Navigation messages are the basic data for navigation and positioning calculations in satellite navigation systems. Nowadays, there is a growing need to evaluate the protocol conformance of navigation messages. In this paper, the evaluation model and the calculation method for protocol conformance of BDS D1 navigation messages are proposed, and the example analysis of actual BDS D1 navigation messages is presented. The evaluation model is described from three aspects, including formatting conformance, verification conformance and quantization unit conformance. Every aspect is considered carefully with calculation and analysis. The results show that the model can evaluate the protocol conformance of BDS D1 navigation messages reasonably.
\end{abstract}

\section{Introduction}

Navigation messages are broadcasted by navigation satellites, containing spatial references and time bases that can be used for navigation and positioning. They are the basic data for navigation and positioning calculations in satellite navigation systems.

Interface Control Document (ICD) is a set of agreed rules for senders and receivers to generate and interpret navigation messages, which is one of the protocols in a satellite navigation system. Only by implementing the same protocol can navigation messages be transmitted properly and effectively. Therefore, it is necessary to evaluate protocol conformance of navigation messages in the process of product development.

At present, research on navigation messages at home and abroad focuses on analyzing the quality of observation data [1-3] and error correcting design [4]. The research on the evaluation model for protocol conformance of navigation messages is still rare.

In this paper, the evaluation model and the calculation method for protocol conformance of BDS D1 navigation messages are proposed, and the example analysis of actual BDS D1 navigation messages is presented.

\section{Evaluation model for protocol conformance of BDS D1 navigation messages}

\subsection{Frame structure of BDS D1 navigation messages}

The frame structure of BDS D1 navigation messages is shown in Fig. 1 [5].

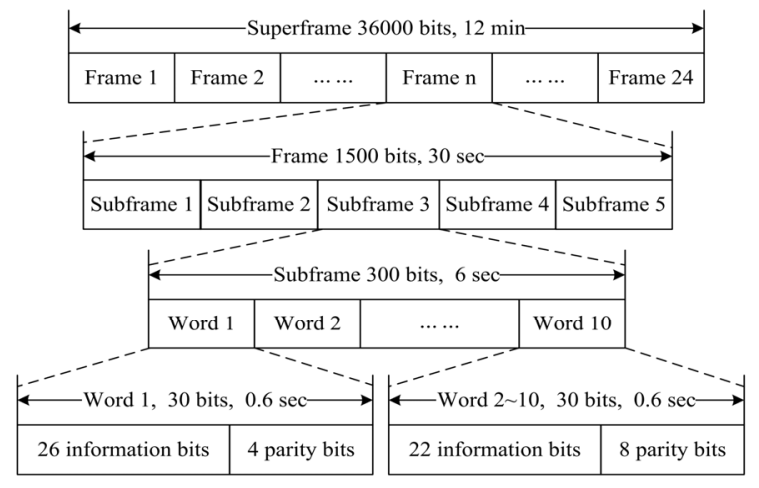

Fig. 1. The frame structure of BDS D1 navigation messages.

Each frame is composed of 5 subframes. Each subframe is composed of 10 words. Each word has 30 bits. Each word consists of navigation message data and parity bits. In the first word of every subframe, the first 15 bits are not encoded and the following 11 bits are encoded in $\mathrm{BCH}(15,11,1)$ for error correction. Each of the other 9 words contains two blocks of $\mathrm{BCH}$ codes with22 information bits in it.

\subsection{Evaluation model for protocol conformance of BDS D1 navigation messages}

Protocol conformance reflects the level of satisfaction of generated navigation messages with ICD.

By studying the requirements in ICD, the evaluation model for protocol conformance of navigation messages is described from three aspects, including formatting

\footnotetext{
* Corresponding author: 1vzhicheng@,chinauninav.com
} 
conformance, verification conformance and quantization unit conformance, as is shown in Fig. 2.

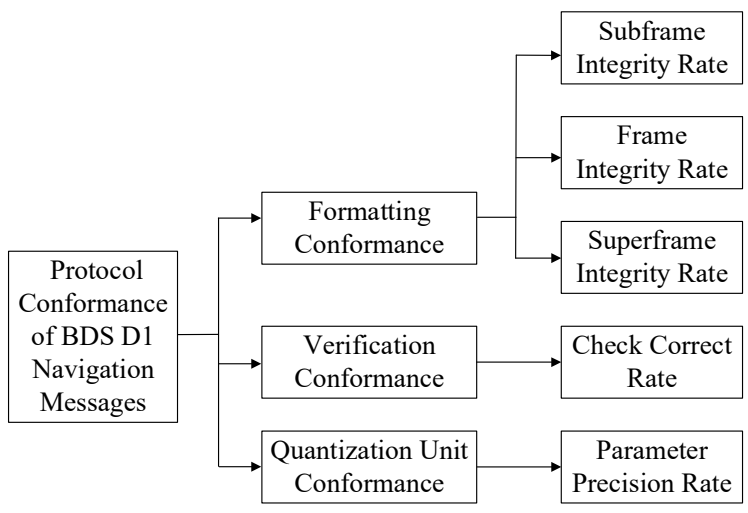

Fig. 2. The evaluation model for protocol conformance of BDS D1 navigation messages.

Formatting conformance is defined as formats of navigation messages comply with the ICD, requiring that messages are complete without any duplication or loss. There are three indicators to describe formatting conformance of navigation messages, including the subframe integrity rate, the frame integrity rate, and the superframe integrity rate.

Verification conformance is defined as check results of error correction decoded navigation messages are correct. The indicator to describe verification conformance of navigation messages is the check correct rate.

Quantization unit conformance is defined as calculated values of parameters solved from navigation messages are close to their true values determined when messages are generated. The indicator to describe quantization unit conformance of navigation messages is the parameter precision rate.

\section{Calculation method for protocol conformance of BDS D1 navigation messages}

\subsection{Calculation method for formatting conformance of BDS D1 navigation messages}

Formatting conformance of BDS D1 navigation messages is described by three indicators, including the subframe integrity rate, the frame integrity rate, and the superframe integrity rate. Since the calculation method of each indicator is the same, this subsection uses the calculation of the subframe integrity rate as an example.

Each subframe of BDS D1 navigation messages consists of 10 words. Each word has 30 bits. The subframe integrity rate of the $i$ th word $I R_{s b f i}$ is calculated as

$$
\left\{\begin{array}{l}
I R_{s b f i}=\sum_{i=1}^{10} \alpha_{i} \cdot \frac{v_{i}}{30} \\
\sum_{i=1}^{10} \alpha_{i}=1
\end{array}\right.
$$

Where $\alpha_{i}$ is the weight of the $i$ th word and $v_{i}$ is the number of valid bits of the $i$ th word. Moreover, $\alpha_{i}$ reflects the importance of the $i$ th word and should be considered carefully.

\subsection{Calculation method for verification conformance of BDS D1 navigation messages}

Verification conformance of BDS D1 navigation messages is described by the check correct rate.

It is necessary to detect whether navigation messages are reversed before verification [6] because of phase ambiguity. It is assumed that this step has already been done at the time of frame synchronization earlier. Then verification of BDS D1 navigation messages can be performed as follows.

Step 1: Take the first 22 information bits from each word of navigation messages. Divide it into two groups of 11 bits each. Then take the other 8 parity bits.

Step 2: According to the BCH $(15,11,1)$ encoding process, calculate the 4 parity bits of each group with 11 information bits respectively. Connect the 2 sets of parity bits into 8 bits. It is worth noting that the one calculated by the first 11 information bits are placed in front, and the others are placed behind.

Step 3: Compare the 8 parity bits calculated in step 2 with the parity bits taken in Step 1. If they are the same, the first 22 information bits from the word of navigation messages are correct.

For the first word of each subframe, the first 15 bits are not encoded, while the following 11 bits are encoded. Therefore, if a word of the navigation message is verified to be the first word of the subframe, then it is feasible to take the 11 bits with number from 16 to 26 and the corresponding 4 parity bits with number from 27 to 30 in Step 1. Then in Step 2, only one encoding calculation is needed to get the 4 parity bits. Compare the two sets of parity bits in Step 3 .

There are 1200 words in a superframe. The check correct rate of a superframe $C C R$ is calculated as

$$
\left\{\begin{array}{l}
C C R=\sum_{j=1}^{1200} \beta_{j} \cdot v_{j} \\
\sum_{j=1}^{1200} \beta_{j}=1
\end{array}\right.
$$

where $\beta_{j}$ is the weight of the $j$ th word, which reflects the importance of the word and should be considered carefully. If the check is correct, $v_{j}$ equals to 1 . Otherwise, it equals to 0 .

\subsection{Calculation method for quantization unit conformance of BDS D1 navigation messages}

Quantization unit conformance of BDS D1 navigation messages is described by the parameter precision rate.

The parameter precision rate $P P R$ is calculated as

$$
P P R=1-\left|\frac{v_{t}-v_{c}}{v_{t}}\right| \times 100 \%
$$


where $v_{c}$ is the calculated value of the parameter solved from the navigation message, and $v_{t}$ is the true value of the parameter determined when the navigation message is generated earlier. Both $v_{c}$ and $v_{t}$ are floating point numbers.

The calculated value of the parameter solved from the navigation message $v_{c}$ can be calculated as

$$
v_{c}=n_{b} \times f
$$

where $n_{b}$ is the number of bits and $f$ is the scale factor.

There are some parameters of navigation messages to be considered, including ephemeris parameters, almanac parameters, ionospheric delay correction model parameters, clock correction parameters, BDT-UTC time offset parameters, and BDT-GNSS time offset parameters. Although the number of parameters is large, the calculation method is the same.

\section{Example analysis for protocol conformance of BDS D1 navigation messages}

\subsection{Example analysis for formatting conformance of BDS D1 navigation messages}

Taking a group of actual BDS D1 navigation messages as example to evaluate the formatting conformance. The data was selected from the International Satellite Navigation System Monitoring and Evaluation Service (iGMAS) in BJF1 station on September 28, 2017 from 0:00.

Since the weights need to be considered carefully, this subsection uses the same weights to calculate. To simplify the expression, this subsection takes the calculation of a superframe and its first subframe and first frame as an example, as is shown in Table 1.

Table 1. Calculation results of the formatting conformance of the actual BDS D1 navigation messages.

\begin{tabular}{|c|c|c|c|c|c|}
\hline PRN & 6 & 7 & 8 & 9 & 10 \\
\hline$I R_{s f f 1}$ & $100.00 \%$ & $100.00 \%$ & $100.00 \%$ & $100.00 \%$ & $100.00 \%$ \\
\hline$I R_{f 1}$ & $100.00 \%$ & $100.00 \%$ & $100.00 \%$ & $100.00 \%$ & $99.87 \%$ \\
\hline$I R_{s p f}$ & $98.92 \%$ & $100.00 \%$ & $100.00 \%$ & $100.00 \%$ & $95.88 \%$ \\
\hline
\end{tabular}

In Table $1, P R N$ is the number of the satellite, $I R_{s b f 1}$ is the subframe integrity rate of the first subframe, $I R_{f 1}$ is the frame integrity rate of the first frame and $I R_{s p f}$ is the superframe integrity rate of the superframe.

For Satellites 7 to 9, they have good formatting conformance. For Satellites 6, a bit loss may occur to other frames, which affects the superframe integrity. For Satellites 10, a bit loss may occur to other subframes of the first frame, which affects the frame integrity and the superframe integrity.

It does not make sense to look at the percentage simply. It is necessary to know where the bit loss occurs so that the correction will reach $100 \%$ of the integrity rate eventually, instead of simply calculating. It is unreasonable that the integrity rate reach $99.9 \%$ while the key bit is wrong.

\subsection{Example analysis for verification conformance of BDS D1 navigation messages}

The data is the same as the previous subsection.

The verification conformance is described by the check correct rate. This subsection uses the same weights to calculate. Table 2 shows the check correct rate of superframes of the actual BDS D1 navigation messages.

Table 2. The check correct rate of the actual BDS D1 navigation messages.

\begin{tabular}{|c|c|c|}
\hline PRN & Error number & $C C R$ \\
\hline 6 & 0 & $100.000000 \%$ \\
\hline 7 & 0 & $100.000000 \%$ \\
\hline 8 & 0 & $100.000000 \%$ \\
\hline 9 & 1 & $99.999071 \%$ \\
\hline 10 & 0 & $100.000000 \%$ \\
\hline
\end{tabular}

In Table 2, $C C R$ is the check correct rate of a superframe with 1200 words. All satellites except Satellites 9 have good verification conformance. For Satellites 9, it indicates that 1 bit error may occur to a word during transmission or baseband processing and the error may be an information bit error or a parity bit error. Where the bit error occurs should be figured out and the error should be corrected.

\subsection{Example analysis for quantization unit conformance of BDS D1 navigation messages}

The data is the same as the previous subsection. Take the ephemeris parameters of Satellite 6 as an example. The result is as shown in Table 3.

Table 3. The parameter precision rate of the ephemeris parameters of Satellite 6

\begin{tabular}{|c|c|c|c|}
\hline Par. & $v_{c}$ & $v_{t}$ & $P P R$ \\
\hline$t_{o e}$ & $1.008000000000 \mathrm{E}+05$ & $1.008000000000 \mathrm{E}+05$ & $100.000000000000 \%$ \\
\hline$\sqrt{A}$ & $6.493335044815 \mathrm{E}+03$ & $6.493335046823 \mathrm{E}+03$ & $99.999999969076 \%$ \\
\hline$e$ & $1.543931746171 \mathrm{E}-04$ & $1.543931746172 \mathrm{E}-04$ & $99.9999999999935 \%$ \\
\hline$\omega$ & $1.963504892640 \mathrm{E}-01$ & $1.963505038930 \mathrm{E}-01$ & $99.999992549548 \%$ \\
\hline$\Delta n$ & $6.255604791828 \mathrm{E}-10$ & $6.259186369169 \mathrm{E}-10$ & $99.942778867254 \%$ \\
\hline$M_{0}$ & $8.003448350212 \mathrm{E}-01$ & $8.003448351777 \mathrm{E}-01$ & $99.999999980446 \%$ \\
\hline$\Omega_{0}$ & $2.130725946031 \mathrm{E}+00$ & $2.130725960318 \mathrm{E}+00$ & $99.999999329477 \%$ \\
\hline$\dot{\Omega}$ & $-1.794837407355 \mathrm{E}-09$ & $-1.794837407356 \mathrm{E}-09$ & $99.999999999944 \%$ \\
\hline$i_{0}$ & $9.607569219780 \mathrm{E}-01$ & $9.607569228630 \mathrm{E}-01$ & $99.999999907885 \%$ \\
\hline$I D O T$ & $-7.549361580454 \mathrm{E}-11$ & $-7.549361580455 \mathrm{E}-11$ & $99.9999999999987 \%$ \\
\hline$C_{u c}$ & $-1.298283539894 \mathrm{E}-05$ & $-1.298494698940 \mathrm{E}-05$ & $99.983738166496 \%$ \\
\hline$C_{u s}$ & $2.650808174265 \mathrm{E}-05$ & $2.650808174266 \mathrm{E}-05$ & $99.99999999999962 \%$ \\
\hline$C_{r c}$ & $-5.815928831984 \mathrm{E}+02$ & $-5.815928831984 \mathrm{E}+02$ & $100.000000000000 \%$ \\
\hline$C_{r s}$ & $-3.932218439467 \mathrm{E}+02$ & $-3.932218439467 \mathrm{E}+02$ & $100.000000000000 \%$ \\
\hline$C_{i c}$ & $-1.298623569240 \mathrm{E}-07$ & $-1.298623569241 \mathrm{E}-07$ & $99.999999999923 \%$ \\
\hline$C_{i s}$ & $-1.133883942967 \mathrm{E}-07$ & $-1.133883942968 \mathrm{E}-07$ & $99.9999999999912 \%$ \\
\hline
\end{tabular}

In Table 3, $v_{c}$ is the calculated value of the parameter solved from the navigation message, and $v_{t}$ is the true value of the parameter determined when the navigation message is generated earlier, and $P P R$ is the parameter precision rate. As can be seen from Table 3, all 
ephemeris parameters have good quantization unit conformance.

\section{Conclusion}

In this paper, the evaluation model for protocol conformance of BDS D1 navigation messages is described from three aspects, including formatting conformance, verification conformance and quantization unit conformance. Each aspect is considered carefully with calculation and analysis. The results show that the model can evaluate the protocol conformance of BDS D1 navigation messages reasonably.

\section{Acknowledgment}

This work is supported by the National Science Foundation of China (Nos. 41604016).

\section{References}

1. F. Bai, Bull. Surv. Map. 6, 72 (2010)

2. W. Yu, W. Dai, Z. Yang, J. Geod. Geodyn., 30, 81 (2010)

3. H. Liu, S. Tang, H. Cui, Geom. Spat. Info. Tech., 1, 5 (2014)

4. S. Lian, L. Yan, Comp. Meas. Cont. 18, 2344 (2010)

5. China Satellite Navigation Office, Beidou Navigation Satellite System Signal In Space Interface Control Document Open Service Signal B3I (Version 1.0), 1, 18 (2018)

6. S. Chen, D. L. Donoho, ION, 55 (1999) 